\title{
WHEELED-ROBOT NAVIGATION WITH VELOCITY UPDATING ON ROUGH TERRAINS
}

\author{
Farid García, Matías Alvarado \\ Centro de Investigación y de Estudios Avanzados-IPN, Departamento de Computación, \\ Av. Instituto Politécnico Nacional 2508, San Pedro Zacatenco, CP 07360, México DF, México \\ farid@computacion.cs.cinvestav.mx, matias@cs.cinvestav.mx
}

Keywords: Autonomous Navigation, Roughness Recognition, Velocity Updating, Wheeled-Robots.

\begin{abstract}
For navigation on outdoor surfaces, usually having different kind of roughness and soft irregularities, this paper proposal is that a wheeled robot combines the gradient method for path planning, alongside it adjusts velocity based on a multi-layer fuzzy neural network; the network integrates information about the roughness and the soft slopes of the terrain to compute the navigation velocity. The implementation is simple and computationally low-cost. The experimental tests show the advantage in the performance of the robot by varying the velocity depending on the terrain features.
\end{abstract}

\section{INTRODUCTION}

Robotic autonomous navigation throughout outdoor terrains is highly complex. Obstacle detection and avoidance for no collision as well as the terrain features information for no slides are both required. Environment data must be quick and accurately processed by the robot's navigation systems for a right displacing. Besides, when information from human remote controllers is not quick available, the autonomous robots should be equipped for convenient reactions, particularly in front of unpredicted circumstances. Actually, by moving on outdoors, the autonomous robot's velocity control regarding the terrain features, beyond the obstacle location and avoidance, it has been few attended and it is a weakness for efficient and safe navigation nowadays.

For wheeled-robots navigation on terrains, it is necessary data about the surface features such that automated safe navigation is ensured. The feature which this work focuses is the surface roughness where the robot moves on. The robot's velocity during real navigation depends on the terrain roughness.

Outdoor autonomous robots are particularly relevant employed for terrain exploration missions. The terrain difficulties of soon system planets -like Mars- to move through soil, rocks and slopes, requires the usage of robots with the highest degree of autonomy to overcome such difficulties. In Earth exploration missions where human lives may be in dangerous circumstances, the autonomous robots are as well required. For instance, search of explosive minas, active volcano craters exploration to determine the eruption risk.

Kelly and Stentz (1998) propose a navigation system for outdoors robots which includes perception, mapping and obstacle avoidance. Regarding the environment perception, Lambert et al. (2008) introduces a probabilistic modelling useful to avoid or to mitigate eventual collisions, which is used for updating a robot braking action. Selekwa et al. (2008) and Ward \& Zelinsky (2000) addressed the navigation and path planning of an autonomous robot which varies the velocity according to the proximity of obstacles detected by infrared sensors.

So far, all the referred works on outdoor autonomous robots do not include in their proposals information about terrain surface roughness during navigation. In this work, two algorithms are implemented for robot autonomous navigation, one for path planning and the other for velocity updating regarding the terrains features. The present proposal is tested with a small wheeled-robot moving over outdoors terrains containing grass, ground, garden sand and soil, as the ones in Figure 1 and Figure 2. It is assumed that the robot can move on slopes with inclination angles less than 15 degrees; otherwise, the slopes are considered as obstacles, thus, the robot wraps them, in order to overcome them.

Surface textures are captured via artificial vision, after image processing the estimation of the terrain 


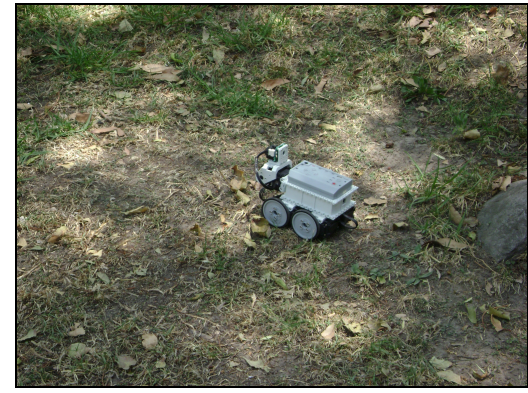

Figure 1: Robot's navigation outdoor surface.

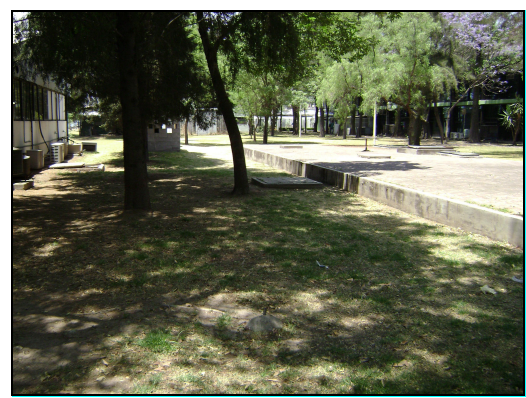

Figure 2: Test environment.

roughness as well as the slopes inclinations are gotten. Then, the algorithms output indicates the velocity the robot can achieve. Bright and uniform lighting during navigation is required to guaranty consistent roughness recognition; therefore the presence of shadows, which treatment is a hard task to pattern recognition (Kahraman and Stegmann, 2006), is out of the scope of this work.

During outdoors navigation, human drivers estimate the convenient vehicle velocity by regarding their previous experience when driving on similar terrain textures. In other words, humans estimate how rough, in average, the terrain is, instead if specific texture details are recognized.

Human drivers that navigate on uneven terrains do not need to know about specific details but on the textures appearance average. The average recognition of ranges of textures as the humans learn is the behave experience to be mimicked and implemented to strength the robots' navigation abilities. The algorithms for path planning differ depending on the type of application, exploration on unknown terrains (Seraji and Howard, 2002), car navigation on roads (Sun et al., 2006), planet exploration (Seraji and Werger, 2007) or indoor navigation (Ward and Zelinsky, 2000), or if the environment is either dynamic (Kim et al., 2007) or static (Wang and Liu, 2005).
For our purpose, the robot moves on the calculated path by adjusting its velocity depending on the terrain features. The path planning algorithm called gradient method, in static environments, recalculates the path in real time whenever an obstacle is found (Konolige, 2000). The gradient method is integrated for our navigation proposal.

The rest of the article is organized as follows: Section 2 summarizes the closest antecedents in the field of autonomous navigation; then, the method and architecture of the fuzzy neural network for speed updating, together with the gradient method for path planning are introduced. Section 3 describes the integration of both algorithms for wheeled-robot navigation, together with the tests and experimental results. Discussion in Section 4, then the paper ends with conclusions in Section 5.

\section{VELOCITY UPDATING BY FUZZY NEURAL NETWORK}

\subsection{Terrain Roughness Recognition}

The classification of terrain roughness has almost no received attention, and just recently is being a bit more attended. For instance, Larson et al. (2005) analysis the terrain roughness by means of spatial discrimination which then is (meta-) classified. Seraji and Howard (2002) assess the navigation strategy with the terrain's features of roughness, slopes and discontinuity. Ishigami et al. (2007) generate a path over a rough terrain with a terrainbased criterion function, and then the robot is controlled so as to move on the chosen path. In Brooks and Iagnemma (2009) do roughness recognition by using artificial vision, so recognition of novel textures is later to off-line recognition training from sample texture. Pereira et al. (2009) plotted maps of terrains incorporating roughness information that is based on the measurement of vibrations occurring in the suspension of the vehicle; this online method can recognize textures at the moment the vehicle passes over them, what is a limitation for velocity updating.

For velocity updating according to the terrain features, our proposal sets to imitate as human beings do. For safe navigation on irregular terrains, the human's velocity estimation is via imprecise but enough surface texture recognition. When a human driver notes a new texture, he uses his experience to estimate how rough the texture is, and then he decides the car driving speed without slide risks. 


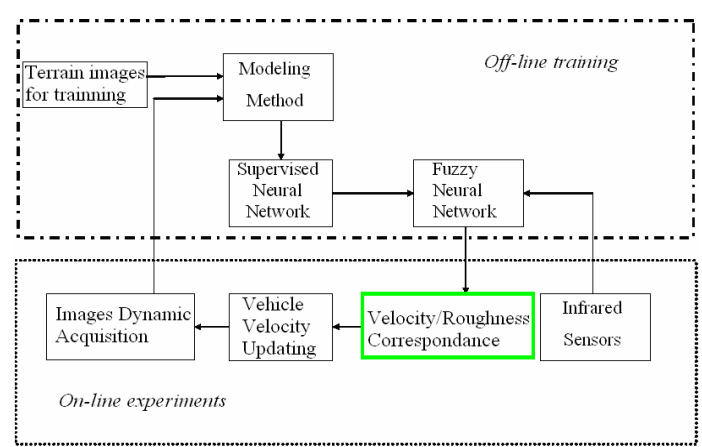

Figure 3: The proposal diagram.

As illustrated in the diagram of Figure 3, in the first step, the terrain's textures are neural-netclustered in a roughness meta-class: a Supervised Neural Network (SNN) classifies textures forming the meta-class; then, a Fuzzy Neural Network (FNN) extends the supervised one, matches each terrain roughness with the corresponding robot velocity meanwhile the robot navigates safely.

Slopes are detected by two infrared sensors. One infrared sensor is located in the frontal part of the robot does parallel ray projection to the robot's motion; the other sensor projects its ray directly to the floor perpendicular to the first sensor. The inclination angle of slopes is computed by trigonometric operations.

The off-line and on-line steps to adapt velocity regarding the terrains roughness and the inclination slopes while navigating are next described:

\section{Roughness identification (off-line training)}

1) Select representative images of the terrain textures, where the robot moves on.

2) Characterize the texture using the Appearance Based Vision method which computes the principal components of the images distribution.

3) Train the SNN with the texture-roughness relationship previously established by the human expert driver.

4) Train the FNN to determine the velocity regarding the texture roughness as well as the inclination angle of slopes, according to an expert driver's directives, make the fuzzy sets and the inferece IF-THEN rules system.

\section{Velocity updating and robot's motion (on-line} steps)

5) Acquisition of terrain images with the robot's camera.
6) The SNN classifies the texture and assigns its roughness, this data is forwarded to the FNN.

7) The FNN inputs are both, the texture roughness and the slope inclination angle (to determine if the robot can pass on the slope, or should move around it).

8) With the texture roughness and slope inclination data, the FNN updates the velocity. The robot's mechanical control system adjust the velocity.

9) The cycle is repeated as the robot moves, and the velocity is cycle updated.

\subsection{The Fuzzy Neural Network}

This section introduces the five-layer fuzzy neural network, whose output sets the velocity the robot can achieve safely. The terrain features recognition followed by the robot velocity tuning is shown in Figure 4. The roughness and slope input data are assessed and then used to adjust the robot's velocity, that is the FNN output data, see Table 1. The FNN first layer inputs are the slope size and the texture roughness, the second layer sets the terms of input membership variables, the third sets the terms of the rule base, the fourth sets the term of output membership variables, and in the fifth one, the output is the robot's velocity. The textures roughness is classified in three fuzzy sets, High $(\mathrm{H})$, Medium (M) and Low (L). The inclination angles of slopes are classified in six fuzzy sets: Plain (Pl), Slightly Plain (SP), Slightly Sloped (SS), Moderato Sloped (MS), High Slope (HS) and Very High (VH). The FNN output values are either: High Velocity (HV), Moderate Velocity (MV), Low Velocity (LW) or Stop (ST). Membership functions of the input and output variables terms denote the corresponding texture roughness, slope inclination and velocity, respectively.

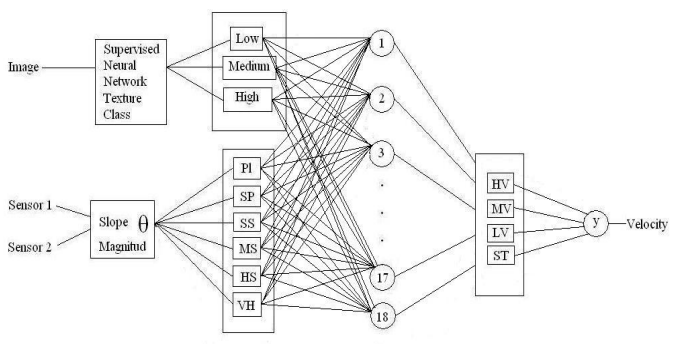

Figure 4: The Fuzzy Neural Network. 
The fuzzy-making procedure maps the crisp input values to the linguistic fuzzy terms with membership values in $[0,1]$. In this work the trapezoid membership functions (MF) for texture variable and the triangle MF for angle variable are respectively used. The FL inference rules governing the input - output relationship are in the Table 1 . Taking $\mathrm{X}, \mathrm{Y}, \mathrm{Z}$ as variables of the respective predicates, the form of inference rules is:

\section{IF Slope angle is X AND Roughness is Y THEN Velocity is Z.}

The de-fuzzy procedure maps the fuzzy output from the inference mechanism to a crisp signal. When the robot finds a slope steeper than the allowed threshold, it stops, and evaluates which movement to make, whose decision concerns to path planning. The gradient method (Konolige, 2000) is integrated to present proposal.

\subsection{The Gradient Method for Path Planning}

The gradient method requires a discrete configuration of the navigation space in which the path cost function is sampled. At each point of the workspace, the gradient method uses a navigation function to generate a gradient field that represents the optimum path to the target point. The gradient of navigation function indicates the path direction with lowest cost, at each point in the navigation space;

Table 1: The velocity updating fuzzy rules.

\begin{tabular}{|c|c|c|c|}
\hline \multirow{2}{*}{$\begin{array}{c}\text { Rule } \\
\text { No. }\end{array}$} & \multicolumn{2}{|c|}{ Input } & Output \\
\cline { 2 - 4 } & Slope angle & Roughness & Velocity \\
\hline 1 & Pl & L & HV \\
\hline 2 & Pl & M & HV \\
\hline 3 & Pl & H & HV \\
\hline 4 & SP & L & MV \\
\hline 5 & SP & M & HV \\
\hline 6 & SP & H & HV \\
\hline 7 & SS & L & MV \\
\hline 8 & SS & M & MV \\
\hline 9 & SS & H & HV \\
\hline 10 & MS & L & LV \\
\hline 11 & MS & M & MV \\
\hline 12 & MS & H & MV \\
\hline 13 & HS & L & LV \\
\hline 14 & HS & M & LV \\
\hline 15 & HS & H & MV \\
\hline 16 & VH & L & ST \\
\hline 17 & VH & M & ST \\
\hline 18 & VH & H & LV \\
\hline
\end{tabular}

this optimum path to the target is continuously calculated, and is determined based on the length and the proximity to obstacles, in addition to any other criteria that may be chosen. By itself, the gradient method can lead the path with the lowest cost in static and completely unknown environments; this method is efficient for real time monitoring the movements of mobile robots equipped with laser beams.

\section{THE NAVIGATION ALGORITHM}

The robot autonomous navigation requires the concurrent operation of path planning and velocity estimation algorithms. The first step is to create a virtual map of the robot navigation space; hence the surface is divided into squares for providing the required detail level of space model. The next step is to calculate the optimal path between initial and goal locations using the gradient method.

After path planning, the texture recognition algorithm is turned on to determine the robot velocity. The roughness surface data in addition to information from sensors that measure the slopes inclination are processed. Hence, the robot receives the instruction to move at the estimated velocity in the prior determined trajectory. If during the trip the sensors detect an obstacle or slopes with inclination greater than 15 degrees, the robot stops and the velocity estimation algorithm is turned off; the obstacle is registered and a new path to the goal location is recalculated. After that, the velocity estimation algorithm is turned on again, and the robot learns to move in the new trajectory at the estimated speed. Otherwise, i.e., if the robot does not find an obstacle on its path, then its speed is updated.

Note that the velocity estimation algorithm is not being executed all the time, but it is turned off when the robot finds an obstacle; at this circumstance, the camera records the obstacle images instead of surface texture. If the velocity estimation algorithm would not be turned off, the velocity would be estimated based on images of the obstacle texture, what is wrong; furthermore, in front of obstacle the robot should overcomes the obstacle with specific movements and the velocity change is irrelevant.

The robot stops when it determines that has reached the goal location. The robot computes its location from the distance it has travelled since the initial location, by using odometry. The following list summarizes the robot navigation steps, see Figure 5: 
1. Create a virtual map of robot space navigation, surface discretization,

2. Define the initial and goal locations of the robot,

3. Compute the path with the gradient method,

4. Artificial vision is turned on for texture recognition,

5. Velocity is estimated with data from textures and slopes of the surface,

6. The robot receives the order to advance along the path at the estimated velocity,

7. The robot velocity is updated when a change in texture is recognized, or if sensors detect a slope on the surface, or if both events occur,

8. If sensors detect an obstacle, or if slope inclination is greater than 15 degrees, then the robot stops and texture recognition algorithm is turned off, return to step 3; Otherwise, velocity is updated, return to step 7 ,

9. The robot stops when it has reached the goal location or destination.

\subsection{Experimental Step}

A car-like rover from the Bioloid robot transformer kit (ROBOTIS, 2010) is used, which uses a processing unit, four servomotors for power transmission to the wheels, two infrared sensors located in the robot front, and a wireless camera on top-front of the robot. The robot dimensions are 9.5 $\mathrm{cm}$ width per $15 \mathrm{~cm}$ length. In these experiments the FNN is trained with terrain textures from images in Figure 1.

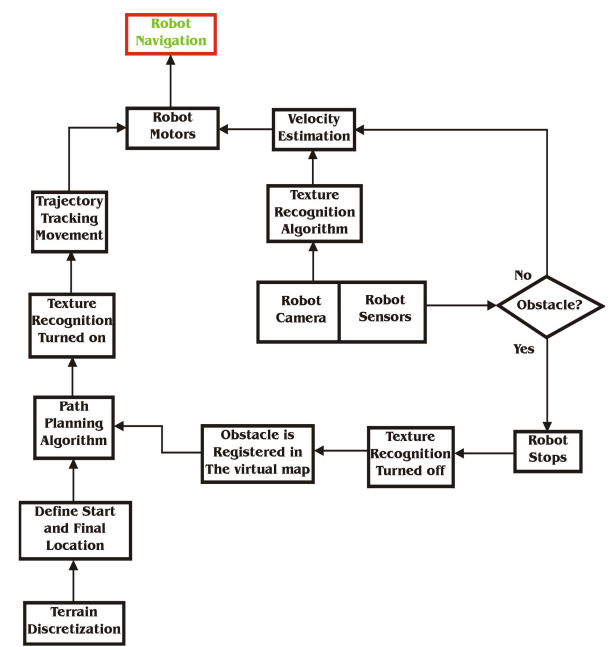

Figure 5: Path Planning and Velocity estimation algorithms running concurrently.
In this platform it is used a personal computer (PC) and the processor of the robot, to form a master-slave architecture, wirelessly communicated. On the PC are implemented and executed the path planning and velocity estimation algorithms. The robot, on one hand, reports to the PC the sensors readings and wirelessly transmits the images captured by the camera. On the other hand, the robot performs the movements in accordance with instructions that the PC communicates it. The experiments are performed in the environment shown in Figure 2, whose area is $2.25 \mathrm{~m}^{2}$, covered with dust, soil, dry leaves, branches and $2 \mathrm{~cm}$-high grass; it contains rocks and small earth-mounds. The goal point is located 2.12 meters in a straight line from the initial location of the robot.

There were conducted 30 tests divided into three parts, using the path planning algorithm. In first part, the tests were performed at medium constant velocity $6.95 \mathrm{~cm} / \mathrm{s}$; in the second part at the maximum velocity the robot can reach $13.88 \mathrm{~cm} / \mathrm{s}$. The other tests were performed with velocity updating, combining path planning and velocity updating algorithms. Table 2 shows the results.

With velocity updating, both the detection of the robot environment and path planning are strengthened. By adjusting the velocity according to surface characteristics, safety increases and/or the travel time of the robot decreases. That is, if it detects that the surface is slippery then the robot slows down, although the robot spends more time to reach the goal location, the probability that the robot has an accident decreases.

\subsection{Results and Navigation Performance}

The common standards criterion to evaluate the performance of robots is (Dai et al., 2007), (Matthies et al., 1995): accurate estimation of the robot location, fast and accurate detection of the robot environment and reliable path planning for moving

Table 2: Autonomous navigation results with and without velocity updating.

\begin{tabular}{|c|c|c|c|}
\hline & \multicolumn{2}{|c|}{ Constant velocity } & $\begin{array}{c}\text { With } \\
\text { velocity } \\
\text { updating }\end{array}$ \\
\hline $\begin{array}{c}\text { Navigation } \\
\text { velocity }\end{array}$ & $6.94 \mathrm{~cm} / \mathrm{s}$ & $\begin{array}{c}13.88 \\
\mathrm{~cm} / \mathrm{s}\end{array}$ & $\begin{array}{c}8.65 \mathrm{~cm} / \mathrm{s} \\
\text { (average } \\
\text { velocity) }\end{array}$ \\
\hline $\begin{array}{c}\text { Navigation } \\
\text { distance }\end{array}$ & $228.5 \mathrm{~cm}$ & $111.29 \mathrm{~cm}$ & $220.33 \mathrm{~cm}$ \\
\hline $\begin{array}{c}\text { Navigation } \\
\text { time }\end{array}$ & $43.10 \mathrm{~s}$ & $19.08 \mathrm{~s}$ & $32.55 \mathrm{~s}$ \\
\hline
\end{tabular}


from one place to another without colliding with obstacles in unknown environments. This is true for this proposal and adds the following:

- The total time it takes the robot to make the route and,

- Comparing the distance travelled by the robot with the straight line distance between the initial and goal locations.

When the robot moves at medium constant velocity $(6.94 \mathrm{~cm} / \mathrm{s})$, it runs $107.72 \%$ of the straight line distance between the initial and goal locations. The travel distance is increased because of the obstacle avoidance and the location estimation errors generated by the slippage of wheels. On the other hand, when the robot moves at maximum constant velocity $(13.88 \mathrm{~cm} / \mathrm{s})$ runs, on average, $52.46 \%$ of the path. The plausible explanation is that when the robot moves at maximum velocity the wheels slip more often and therefore the location estimation of the robot becomes very imprecise. With velocity updating the robot travels the $103.86 \%$ of the straight line distance between the initial and goal locations. In this case the distance is less than with medium constant velocity because the wheel slippage is less frequent, see Table 3. By updating the velocity the robot moves slower in areas that favour the slippage of wheels, for instance loose soil. Because of there are fewer slippages, the location estimation of the robot is more accurate and consequently the robot approaches the goal location.

The navigation time with velocity updating is $32.41 \%$ less and $41.38 \%$ higher than medium and maximum constant velocity, respectively. It is noted that with medium constant velocity the robot travels a path with good accuracy but spends more time doing the travelling. With maximum constant velocity the travelling is fast but the accuracy to traverse the path is very bad. With velocity updating performance is improved because the precision of the robot in the travelling of the trajectory is good and is performed in less time, i.e., the robot moves at optimum velocity, depending on surface characteristics, avoiding wheel slippage. With our approach the average velocity represents $62.13 \%$ of the maximum velocity the robot can reach.

\section{DISCUSSION}

Within the present approach, the robot moves on surfaces with different kinds of textures, to make navigation more versatile than such related works. The Martian surface can be considered as a special case of our approach because these surfaces are
Table 3: Percentage of travelled distances, with maximum and medium constant velocity; and with velocity updating.

\begin{tabular}{|c|c|c|c|}
\hline \multirow{2}{*}{} & \multicolumn{2}{|c|}{ Constant Velocity } & $\begin{array}{c}\text { With } \\
\text { velocity } \\
\text { updating }\end{array}$ \\
\cline { 2 - 3 } & Medium & Maximum & \\
\hline $\begin{array}{c}\text { Percentage } \\
\text { of distance } \\
\text { travelled }\end{array}$ & $107.72 \%$ & $52.46 \%$ & $103.86 \%$ \\
\hline
\end{tabular}

covered with sand and rocks, i.e., there is only one type of roughness. Actually, for the purpose of autonomous navigation on rough terrains is not a requisite to recognize textures at a high detail level. The high precision methods on details recognition are not the adequate but failed for supporting robots navigation -strongly some times. In addition, the detailed recognition of surfaces is computationally expensive, but a low consume of resources is recommended through autonomous navigation.

Our approach can be improved on the location estimation of the robot. So far, it has been used odometry only to calculate the robot location. Most of the works, if not all, that employ odometry use other tools to estimate the location of the robot such as electronic compasses (Seraji and Werger, 2007), sonar sensors (Dai et al., 2007), GPS (Matthies et al., 1995), among others. However, velocity updating reduces wheel slippage and the drift errors are small or occur less often.

On the other hand, the proposed algorithms are not limited to be applied to small vehicles. They can be extrapolated to other vehicles, depending on the particular characteristics, which define the appropriate rules of the vehicle operation. The algorithm is scalable to different vehicles by using as parameters their particular characteristics, such as weight, size and motor power, tires material and tire tread, among others.

In this proposal we claim that for velocity updating the experience of human drivers is mimicking by using the inference system of the fuzzy neural network, which model the operation of the vehicle based on the driver experience.

There are works that model the vehicles driving with differential equations (Nakamura et al., 2007), (Kim et al., 2008), (Ward and Iagnemma, 2008). But this approach is difficult because, in general, differential equations are nonlinear, and their solution is hard to obtain.

Within the algorithms testing, we have simulated the path of a truck. These tests consisted of placing a camera on the roof of the truck. The truck runs on various types of textures. During the truck trips, the camera recorded from a similar driver's visual field 
the crossed surfaces. Then, texture images are extracted from video recordings, which are processed by the algorithms.

The velocity updating results are encouraging. To process the $480 \times 640$-pixel images with the microprocessor Centrino Core 2 Duo at $2 \mathrm{GHz}$ and $1.99 \mathrm{~Gb}$ RAM, the algorithms time spent is small, 0.3 seconds. It leads to conclude that vehicles with these computer capacities have enough time to react or to break on the next 5 meters, as soon as they are moving at $60 \mathrm{~km} / \mathrm{hr}$, which is a car maximum velocity in the city, and a standard speed on principal roads.

\section{CONCLUSIONS}

In this paper a proposal for wheeled robot navigation on outdoor surfaces with different kind of roughness and soft irregularities is presented. The robot integrates the path planning gradient method with a multi-layer fuzzy neural network in order to adjust velocity, by regarding the roughness and the slopes of the terrain. The artificial vision implementation is computationally low-cost. Wheeled-robot navigation becomes more efficient and safe because of the velocity updating. That is because, whenever the robots navigates, the velocity is updated by regarding the terrains characteristics, the wheel slippage is significant reduced, hence improving, the precision to achieve the goal location as well as the navigation time; thereafter, the risk that the robot suffers an accident is also decreased. On the opposite, without velocity updating it becomes more difficult the goal location approach as reported results show.

\section{ACKNOWLEDGMENT}

The authors would like to thank the financial support of CINVESTAV-IPN, Centro de Investigación y de Estudios Avanzados del Instituto Politécnico Nacional. As well Farid García, scholarship no. 207029, would like to thank the financial support of CONACyT, Consejo Nacional de Ciencia y Tecnología.

\section{REFERENCES}

Brooks, C.A. and Iagnemma, K. (2009). Visual Detection of Novel Terrain via Two-Class Classification. In:
Proceedings of the 2009 ACM Symposium on Applied Computing, 1145-1150.

Dai, X., Zhang, H. and Shi, Y. (2007). Autonomous Navigation for Wheeled Mobile Robots-A Survey. In: Second International Conference on Innovative Computing, Information and Control, September 5-7, Kumamoto, Japan, 2207-2210.

Ishigami, G., Nagatani, K. and Yoshida, K. (2007). Path Planning for Planetary Exploration Rovers and Its Evaluation Based on Wheel Slip Dynamics. In: IEEE International Conference on Robotics and Automation, April 10-14, Roma, Italy, 2361-2366.

Kahraman, F. and Stegmann, M.B. (2006). Towards Illumination-Invariant Localization of Faces Using Active Appearance Models. In: 7th Nordic Signal Processing Symposium, June 7-9, Rejkjavik, Iceland, 4.

Kelly, A. and Stentz, A. (1998). Rough Terrain Autonomous Mobility - Part 2: An Active Vision, Predictive Control Approach. Autonomous Robots, 5(2), 163-198.

Kim, P.G., Park, C.G., Jong, Y.H., Yun, J.H., Mo, E.J., Kim, C.S., Jie, M.S., Hwang, S.C. and Lee, K.W. (2007). Obstacle Avoidance of a Mobile Robot Using Vision System and Ultrasonic Sensor. In: Third International Conference on Intelligent Computing, August 21-24, Qingdao, China, 545-453.

Kim, Y.C., Min, K.D., Yun, K.H., Byun, Y.S. and Mok, J.K. (2008). Steering Control for Lateral Guidance for an All Wheel Steered Vehicle. In: International Conference on Control, Automation and Systems. October 14-17, Seoul, Korea, 24-28.

Konolige, K. (2000). A Gradient Method for Realtime Robot Control. In: Proceedings of the IEEE/RSJ International Conference on Intelligent Robots and Systems. October 31 - November 5, Takamatsu, Japan, 639-646.

Lambert, A., Gruyer, D., Pierre, G.S. and Ndjeng, A.N. (2008). Collision Probability Assessment for Speed Control. In: $11^{\text {th }}$ International IEEE Conference on Intelligent Transportation Systems. October 12-15, Beijing, China, 1043-1048.

Larson, A.C., Voyles, R.M. and Demir, G.K. (2005). Terrain Classification Using Weakly-Structured Vehicle/Terrain Interaction. Autonomous Robots, 19(1), 41-52.

Nakamura, S., Faragalli, M., Mizukami, N., Nakatani, I., Kunii, Y. And Kubota, T. (2007). Wheeled Robot with Movable Center of Mass for Traversing over Rough Terrain. In: Proceedings of the IEEE/RSJ International Conference on Intelligent Robots and Systems. October 29 - November 2, 1228-1233.

Matthies, L., Gat, E., Harrison, R., Wilcox, B., Volpe, R. and Litwin, T. (1995). Mars Microrover Navigation: Performance Evaluation and Enhancement. Autonomous Robots, 2(4), 291-311.

Pereira, G.A.S., Pimenta, L.C.A., Chaimowicz, L., Fonseca, A.F., de Almeida, D.S.C., Correa, L.Q., Mesquita, R.C. and Campos, F.M. (2009). Robot Navigation in Multi-Terrain Outdoor Environments. 
International Journal of Robotic Research, 28(6), 685-

700.

ROBOTIS Co., (2010). http://www.robotis.com.

Selekwa, M.F., Dunlap, D.D., Shi, D. and Collins, E.G.

(2008). Robot Navigation in Very Cluttered Environments by Preference-Based Fuzzy Behaviors. Robotics and Autonomous Systems, 53(3), 231-246.

Seraji, H. and Howard, A. (2002). Behavior-Based Robot Navigation on Challenging Terrain: A Fuzzy Logic Approach. IEEE Transactions on Robotics and Automation, 18(3), 308-321.

Seraji, H. and Werger, B. (2007). Theory and Experiments in SmartNav Rover Navigation. Autonomous Robots, 22(2), 165-182.

Sun, Z., Bebis, G. and Miller, R. (2006). On-Road Vehicle Detection: A Review. IEEE Transaction on Pattern Analysis and Machine Intelligence, 28(5), 694-711.

Wang, M. and Liu, J.N.K. (2005). Behavior-Blind GoalOriented Robot Navigation by Fuzzy Logic. In: Proceedings of Knowledge-Based Intelligent Information and Engineering Systems, 686-692.

Ward, C.C. and Iagnemma, K. (2008). A Dynamic-ModelBased Wheel Slip Detector for Mobile Robots on Outdoor Terrain. IEEE Transactions on Robotics, 24(4), 821-831.

Ward, K. and Zelinsky, A. (2000). Acquiring Mobile Robot Behaviors by Learning Trajectory Velocities. Autonomous Robots, 9(2), 113-133. 